\title{
Clinical Courses and Characteristics of Residents During a SARS-CoV-2 Outbreak at a Central Indiana Skilled Nursing Facility
}

\author{
Lauren Albert ${ }^{1}$, Kristi Lieb ${ }^{1}$, Laramie Mack ${ }^{2}$, Kathleen Unroe ${ }^{1,2}$ \\ ${ }^{1}$ Indiana University School of Medicine; ${ }^{2}$ Regenstrief Institute, Center for Aging Research
}

Background/Objective: Older adults such as skilled nursing facility residents have increased risk of serious SARS-CoV-2 infection and comprise a large proportion of the COVID-19 pandemic's deceased-the US Centers for Medicare \& Medicaid Services report 232,831 cases and 38,518 resident deaths to date. Recent case reports reveal, as in other diseases, older adults may experience atypical symptomology, complicating identification of ill residents and efforts to slow transmission. While a few facility outbreaks have been characterized epidemiologically, little research exists regarding clinical timelines and trajectories which residents experience during COVID-19 illness.

Methods: From May 9, 2020-June 1, 2020, daily notes on each COVID-19 positive resident's status $(n=69)$ were taken by the medical director of a central Indiana nursing facility. Combined with a retrospective resident chart review of this same period, these notes were examined for COVID-19 infection symptoms and illness timelines to descriptively categorize a number of common illness trajectories and symptoms seen in residents with SARS-CoV-2 infection.

Results: Residents fit four descriptive clinical timelines: concurrent symptom load with quick death (Avg 5.6 days) $(n=5)$, accumulating symptom load with gradual decline (Avg. 13.9 days) $(n=9)$, prolonged active symptom load with periods of stabilization and symptom reoccurrence $(n=42)$, and asymptomatic or atypical symptom load $(n=12)$. Most common symptoms were fever, hypoxia, anorexia, and fatigue/malaise. Of the 14 residents who died ( $20.3 \%$ of infected), 8 died in the facility and 6 died in the hospital.

Conclusion and Implications: This retrospective case study adds to literature describing the presentation and symptomology of SARS-CoV-2 infection in residents of skilled nursing facilities and aids efforts to evaluate resident presentation, prognosis, and disease course. Robust descriptions of expected clinical courses may support realistic expectations of disease progression for residents and their family members experiencing future outbreaks. 